\title{
O Uso do Facebook como Ambiente Virtual de Aprendizagem no Ensino de Química Orgânica em Língua Inglesa
}

Julie Charline Siqueira de Oliveira - Instituto Federal de Educação, Ciência e Tecnologia do Rio Grande do Sul (IFRS) - juliecharline@ gmail.com

Aline Grunewald Nichele - Instituto Federal de Educação, Ciência e Tecnologia do Rio Grande do Sul (IFRS) - aline.nichele@ poa.ifrs.edu.br

Resumo: Essa pesquisa teve como objetivo investigar como o Facebook pode ser utilizado como um Ambiente Virtual de Aprendizagem (AVA) para o ensino de Química Orgânica em Língua Inglesa. Inicialmente foi realizada uma revisão de literatura de trabalhos acadêmicos que focaram no uso desta rede social no contexto educacional, apresentando alguns exemplos do que já existe e destacando algumas estratégias de ensino e aprendizagem praticadas com as Tecnologias da Informação e Comunicação (TIC). Logo após essa pesquisa esteve direcionada a conceber estratégias de ensino e aprendizagem mediadas pelo Facebook, utilizando como base os conhecimentos oriundos da revisão de literatura. Uma proposta de aulas digitais a serem conduzidas via Facebook como AVA foi concebida e aplicada em turma de um curso Técnico Subsequente em Química do Instituto Federal de Educação, Ciência e Tecnologia do Rio Grande do Sul (IFRS). Os participantes avaliaram a contribuição do Facebook como AVA por meio de questionários, que permitiram identificar também o perfil tecnológico desses participantes. Por fim foi possível conhecer o potencial educacional e a viabilidade da utilização do Facebook como AVA, pois 92,3\% dos participantes da pesquisa avaliaram positivamente as atividades desenvolvidas. Neste artigo apresentamos o relato dessa experiência.

Palavras-chave: AVA. Facebook. Química.

\section{Using Facebook as a Learning Management System to teach Organic Chemistry in English Language}

\begin{abstract}
The objective of this research was to investigate how Facebook can be used as a Learning Management System (LMS) to teach Organic Chemistry in English Language. At the beginning, a research on academical studies that already used Facebook as an LMS was made, showing different learning and teaching strategies using Information and Communication Technologies (ICT). After that, this study was dedicated to design teaching and learning strategies using as basis the literature review's knowledge. A proposal of digital classes developed through Facebook was conceived and applied in a Subsequent Technical Chemistry Course at the Federal Institute of Education, Science and Technology of Rio Grande do Sul, Brazil. The participants then evaluated the Facebook's contribution answering questionnaires, that also allowed to identify the technological profile of these participants. Their answers made Facebook's viability as an LMS very clear, considering that $92,3 \%$ of the students evaluated positively the activities developed. In this paper we report this experience.
\end{abstract}

Keywords: Chemistry. Facebook. LMS.

V. $17 \mathrm{~N}^{\circ}$ 3, dezembro, 2019

DOI: 


\section{Introdução}

As Tecnologias de Informação e Comunicação (TIC) exercem um papel cada vez mais importante na sociedade, elas estão presentes no nosso dia a dia, no trabalho, nas relações interpessoais, na escola e também as utilizamos nos nossos momentos de lazer. Elas trouxeram muitas facilidades e também alguns desafios.

Assim como nossas atividades cotidianas mudaram com as TIC, as estratégias de ensino e aprendizagem também tendem a mudar e a acompanhar essas transformações tecnológicas. Uma vez que as pessoas têm mais acesso à tecnologia e a novos meios de comunicação, a troca de conhecimento e os processos educativos tendem a transformar-se e aproximar-se da realidade das pessoas.

A sociedade mudou sua atitude geral com o advento das novas tecnologias, houve implicações culturais com a virtualização das informações, influenciando em diferentes aspectos das nossas vidas. Castells (1999) também defende a importância de analisarmos a revolução tecnológica por sua influência em todas as esferas da sociedade e sua complexidade "[...]devido a sua penetrabilidade em todas as esferas da sociedade humana, a revolução da tecnologia da informação será meu ponto inicial para analisar a complexidade da nova economia, sociedade e cultura em formação" (Castells, 1999, p. 43).

Além disso, ele considera o impacto das tecnologias na sociedade e como esses temas não podem ser estudados em separado "o dilema do determinismo tecnológico é, provavelmente, um dilema infundado, dado que a tecnologia é a sociedade, e a sociedade não pode ser entendida ou representada sem suas ferramentas tecnológicas" (Castells, 1999, p. 43). Diante deste "determinismo tecnológico", onde sociedade e tecnologia não podem ser entendidas ou estudadas em separado, a temática referente às TIC no campo educacional ganha importância e está reconhecidamente presente nas pesquisas em educação, tendo consenso entre os pesquisadores da área de que as TIC têm um grande potencial de colaborar positivamente nos processos de ensino e aprendizagem.

As redes sociais, estruturas sociais virtuais compostas por pessoas e/ou organizações conectadas por um ou vários tipos de relações, que partilham valores e objetivos comuns na internet, são exemplos desses avanços tecnológicos.

$\mathrm{Na}$ educação, as redes sociais podem ser utilizadas nos processos de ensino e aprendizagem. Campos e Sampaio (2017) constataram que os estudantes participam diariamente e mais assiduamente das redes sociais - como o Facebook, Twitter, Instagram do que das plataformas tradicionais de suporte ao ensino e aprendizagem.

Em tentativas de trazer para o âmbito educacional as TIC que possam estimular os estudantes a participarem com maior interação nos processos de ensino e aprendizagem, o Facebook é citado em diversos estudos (Schroeder; Greenbowe, 2009; Catão, 2014; Porto; Santos, 2014; Campos; Sampaio, 2017; Silva; Martins Junior, 2017) como uma opção bem aceita pelos estudantes. Porto e Santos (2014) salientam a facilidade de comunicação trazida por esta rede social, pois aproxima pessoas e funciona como uma rede virtual de colaboração, tanto no âmbito pessoal quanto profissional. Essas autoras relataram que "ao interagirmos com colegas de outras universidades, notamos que a mídia social Facebook se destacava como meio material e ou intelectual em diversos projetos de pesquisa e formação de professores na cibercultura" (Porto; Santos, 2014, p.15). 
Os Ambientes Virtuais de Aprendizagem (AVA) foram desenvolvidos para ajudar estudantes, professores e tutores nos processos de ensino e aprendizagem presenciais ou a distância ${ }^{1}$, papel que pode ser desempenhado pelo Facebook.

Trazendo as experiências com o uso do Facebook como AVA para o campo das Ciências, Schroeder e Greenbowe (2009) criaram uma comunidade online sobre química orgânica na Universidade Estadual de Iowa, nos Estados Unidos. Levando em consideração que as redes sociais têm grande influência nas vidas dos estudantes; o objetivo inicial do estudo era investigar se os estudantes iriam discutir conceitos de Química com mais frequência por meio do Facebook do que eles faziam no WebCT (o AVA utilizado pela instituição de ensino). Eles escolheram o Facebook por acreditar que facilitaria a comunicação sobre ideias científicas, considerando que os estudantes já utilizavam a rede social por um período de tempo considerável durante a semana. Os estudantes poderiam utilizar a rede social para conversarem entre si e também com o instrutor do laboratório, com os assistentes do professor e com o bibliotecário do projeto por meio de um grupo fechado. Um dos aspectos positivos do uso do Facebook foi a facilidade que os estudantes encontraram para compartilhar fotos, de forma complementar aos textos postados no grupo. Esta função permitiu a qualquer participante responder comentários, explicações ou observações com diagramas, figuras e/ou gráficos relevantes. Ao final do estudo observaram que o número de postagens no Facebook foi aproximadamente $400 \%$ maior do que no WebCT, e essas postagens geraram tópicos mais complexos e comentários mais detalhados. Outro aspecto observado foi que as discussões no fórum do WebCT cessaram ao longo do semestre, enquanto no Facebook se mantiveram constantes. Estas características se devem ao fato de os alunos já estarem acessando o Facebook para uso pessoal e acabavam por checar as postagens no grupo enquanto acessavam a rede social por outras razões.

Nesse contexto, essa pesquisa teve como objetivo investigar como o Facebook pode ser utilizado como um AVA para o ensino de Química Orgânica em Língua Inglesa. Para isso, o estudo do uso do Facebook como um AVA por meio de aulas digitais transdisciplinares com conteúdo de química orgânica ministrados em língua inglesa envolveu a concepção de aulas digitais, bem como a avaliação pelos estudantes da aula realizada por meio do Facebook.

\section{Metodologia da Pesquisa}

A etapa inicial dessa pesquisa se debruçou a conhecer o potencial educacional do Facebook. Para tanto, foi realizada uma revisão de literatura de trabalhos acadêmicos disponíveis que focassem o uso desta rede social no contexto educacional. A busca se deu por meio do Portal de Periódicos da Capes abrangendo o período de 2004 a 2019 (o início desse período foi definido considerando-se que o Facebook foi lançado em 2004) utilizando as palavras-chave "Facebook" e "Ensino", "Facebook" e "Educação", "Facebook" e "AVA", "Facebook" and "Teaching", "Facebook" and "Education", "Facebook" and "Learning Platform". A seleção dos trabalhos acadêmicos encontrados ocorreu a partir da leitura dos resumos dos mesmos. Aqueles que foram considerados mais relevantes para apoiar a revisão de literatura do ponto de vista dos objetivos desta pesquisa foram lidos na íntegra. Na etapa seguinte, essa pesquisa esteve direcionada a conceber estratégias de ensino e aprendizagem

${ }^{1}$ EDOOLS, O que é AVA? Disponível em: https://www.edools.com/faq/o-que-e-ava/ Acesso em: 12 abr. 2019. 
apoiadas no Facebook, utilizando como base os conhecimentos oriundos da revisão de literatura. Inicialmente foi elaborado um plano de aula e, a partir dele, um grupo fechado intitulado "Organic Chemistry - IFRS" foi criado, com as propostas de intervenção.

Para avaliação das propostas de intervenção a pesquisa tomou forma de um estudo de caso, com a análise da aceitação e participação nessas atividades por parte dos estudantes. $\mathrm{O}$ estudo de caso consistiu na aplicação das aulas digitais de química orgânica em língua inglesa e permitiu a coleta de informações acerca do grupo de estudantes participantes.

Como instrumentos para coleta de dados da etapa de aplicação das aulas digitais, foram utilizados dois questionários compostos por questões abertas e fechadas. A pesquisa foi aplicada a 13 estudantes, todos maiores de 18 anos, do segundo semestre de um curso Técnico Subsequente em Química. As atividades ocorreram em laboratório de informática da instituição de ensino e foram previamente planejadas de modo a iniciar com palavras cognatas (palavras com escrita semelhante ao português e mesmo significado) e irem avançando no vocabulário de língua inglesa. Para cada atividade foi disponibilizado também um material de apoio em língua inglesa.

O primeiro questionário foi aplicado antes da participação nas aulas digitais e permitiu identificar o perfil tecnológico dos participantes. O segundo foi aplicado logo após a participação em cada aula digital e permitiu avaliar a contribuição do Facebook como AVA.

Para a análise dos dados das questões fechadas foi efetuada a tabulação simples, e para a análise dos dados das questões abertas foi utilizada a análise textual discursiva (ATD) (Moraes; Galiazzi, 2006).

\section{Resultados}

A escolha em estudar o uso do Facebook como AVA deu-se pela ampla utilização; por não ser necessário cadastrar os estudantes na plataforma, por oferecer acesso gratuito e por possuir uma interface amigável e intuitiva, já bem conhecida por professores e estudantes. Diante disso, esta pesquisa buscou contribuir nos processos de ensino e aprendizagem da disciplina de química orgânica, por meio da utilização do Facebook, desempenhando o papel de um AVA, onde foram criadas propostas de intervenção transdisciplinares (na forma de aulas digitais) no âmbito educacional, engajando os estudantes em atividades planejadas e compartilhadas em um ambiente que lhes é familiar, desenvolvendo conteúdos de química orgânica em língua inglesa.

A seguir são apresentados o perfil tecnológico dos participantes, a descrição de duas aulas digitais utilizando o Facebook como AVA e a avaliação dessas atividades pelos estudantes participantes das atividades.

Conhecer o perfil tecnológico dos participantes foi importante para saber como conduzir as propostas de intervenção por meio do Facebook e identificar a necessidade ou não de uma formação prévia acerca da utilização da rede social e suas funcionalidades.

Dos 13 participantes da pesquisa, $11(84,6 \%)$ eram do sexo feminino e $2(15,4 \%)$ do sexo masculino. Em relação à faixa etária, 69,2\% (9 estudantes) tinham entre 18 a 24 anos, 23,1\% (3 estudantes) tinham entre 25 a 34 anos, 7,7\% (1 estudante) tinha entre 35 a 44 anos. Em relação à frequência dos acessos ao Facebook, 84,6\% (11 dos estudantes) declararam utilizar a rede social diariamente.

Sobre o local de onde esses acessos são realizados, 11 estudantes participantes da pesquisa declararam utilizar dispositivos móveis, o equivalente a 84,6\%. 
Quando perguntados sobre quais atividades os estudantes realizavam por meio da rede social, 38,5\% (5 estudantes) responderam que utilizam principalmente para acompanhar notícias e novidades de pessoas e empresas, 30,8\% (4 estudantes) para manter contato com amigos e familiares e outros 30,8\% (4 estudantes) para manter contato com colegas de aula ou de trabalho, demonstrando assim o potencial do Facebook para facilitar a comunicação entre estudantes e professores. Quanto à participação em grupos na rede social, $11(84,6 \%)$ dos estudantes disseram já participar e apenas $2(15,4 \%)$ não participavam.

A forte presença deste grupo de estudantes no Facebook reafirma a escolha em estudar o uso desta rede social como AVA, todos já estavam familiarizados com as funcionalidades oferecidas, não sendo necessário um treinamento prévio nem os cadastrar em uma nova plataforma de suporte ao ensino e aprendizagem, conforme já havia sido observado por Campos e Sampaio (2017).

Após responderem o questionário sobre o perfil tecnológico, os estudantes iniciaram as aulas digitais conduzidas via grupo fechado do Facebook.

A atividade 1 consistia na apreciação do conteúdo de uma imagem sobre acidificação dos oceanos e sinalização química (Ocean Acidification and Chemical Signaling) (Figura 1).

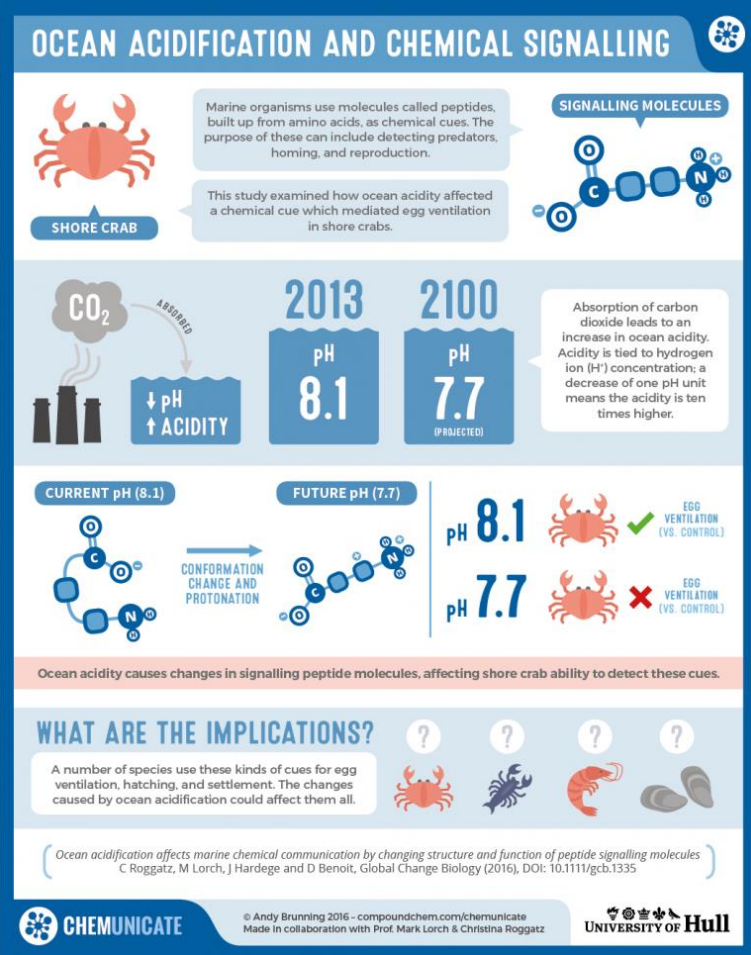

Figura 1 - Atividade Sobre Acidificação dos Oceanos.

Fonte: https://www.theguardian.com/science/blog/2016/jun/29/thanks-to-co2-emissions-the-smellof-the-sea-is-changing-ocean-acidification

A partir do conteúdo da Figura 1 os estudantes foram provocados a trabalhar com o vocabulário abordado, foram postadas perguntas (em língua inglesa, "What causes ocean acidification?") no espaço dos comentários do texto, como forma de instigá-los a buscar as respostas. Para responder, os estudantes puderam optar entre utilizar a língua inglesa ou a língua portuguesa, conforme se sentissem mais confortáveis, e todos responderam em português. Foi elaborado também um glossário colaborativo (Figura 2), com a finalidade de mapear o vocabulário desconhecido por eles, por meio do qual a pesquisadora e os estudantes 
preencheram a definição e a tradução das palavras que emergiam. Os estudantes tiveram a liberdade de utilizar o Google Translate para pesquisar.

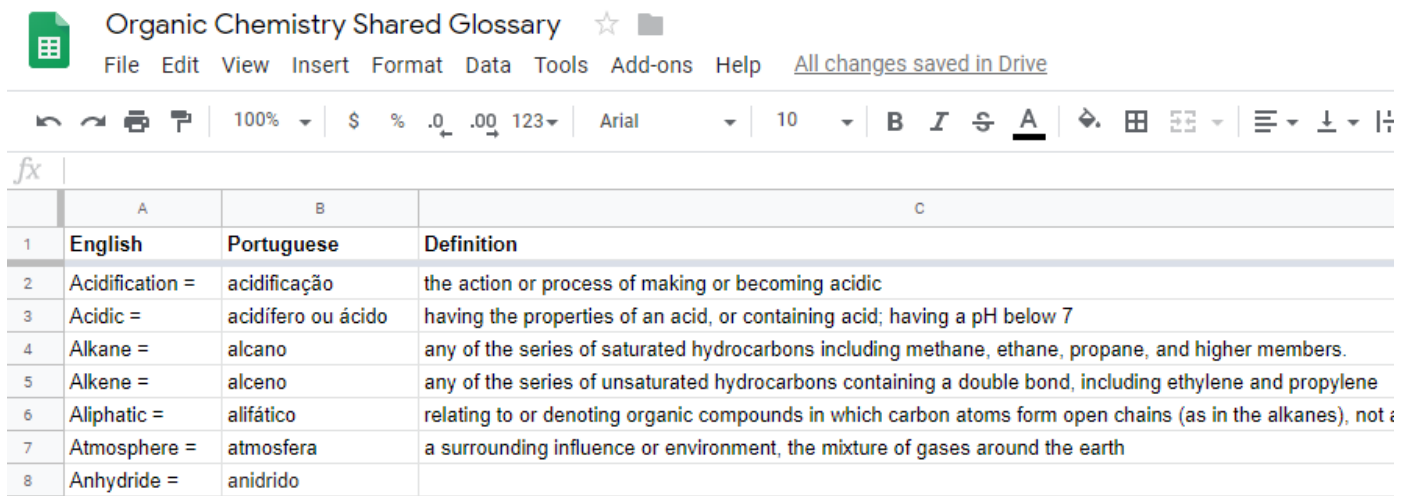

Figura 2 - Glossário Colaborativo.

Fonte: Glossário construído por meio do Google Sheets.

Os estudantes participaram ativamente das tarefas propostas, responderam todas as perguntas postadas nos comentários e ajudaram a completar o glossário colaborativo. A busca pelas traduções das palavras desconhecidas gerou interação entre eles, pois antes de procurar no mecanismo de busca e tradução, eles interagiam com os colegas buscando o significado.

Após o encerramento da "Aula Digital 1" a pesquisadora postou novas atividades no grupo (para a aula 2) e observou que 8 alunos entraram no grupo fora do horário escolar. Esta característica se deve ao fato de os alunos já estarem acessando o Facebook para uso pessoal e acabaram por verificar as postagens no grupo enquanto acessavam a rede social por outras razões, conforme constatado no estudo de Schroeder e Greenbowe (2009).

A "Aula Digital 2" iniciou com a atividade: "Functional Groups in Organic Chemistry" (Figura 3), que consistia na leitura e pronúncia (em língua inglesa) dos nomes das funções orgânicas. Os estudantes utilizaram o Google Translate para pesquisar o significado das palavras que não conheciam e também para verificar a pronúncia. Neste momento foram distribuídos fones de ouvido para que os sons não se misturassem e atrapalhassem os colegas. Novamente as palavras desconhecidas foram colocadas no glossário colaborativo (Figura 2).

Após a apropriação e reconhecimento das funções orgânicas e da leitura texto complementar, os estudantes realizaram um teste que consistia em desenhar as funções orgânicas solicitadas e identificar as que já estavam desenhadas. Ao finalizar, eles postaram as imagens dos testes no grupo. Na segunda parte da aula 2 os participantes assistiram a um vídeo sobre álcool como combustível - Alcohol Fuel (The Audiopedia ${ }^{3}$ ), que continha o áudio e o texto para que os estudantes acompanhassem a leitura e pronúncia de cada palavra. Para esta atividade eles utilizaram os fones de ouvido, considerando que cada estudante tinha a liberdade de assistir o vídeo a seu tempo e voltar o texto caso sentisse necessidade.

2 ASHENHURST, James. Meet the most important Functional Groups. Disponível em: https://www.masterorganicchemistry.com/2010/10/06/functional-groups-organic-chemistry/. Acesso em: 16 abr. 2019.

3 THE AUDIOPEDIA. Alcohol Fuel. Disponível em: https://www.youtube.com/watch?v=jgXxs9wgGSA. Acesso em: 16 abr. 2019.

V. $17 \mathrm{~N}^{\circ} 3$, dezembro, 2019 RENOTE

DOI: 


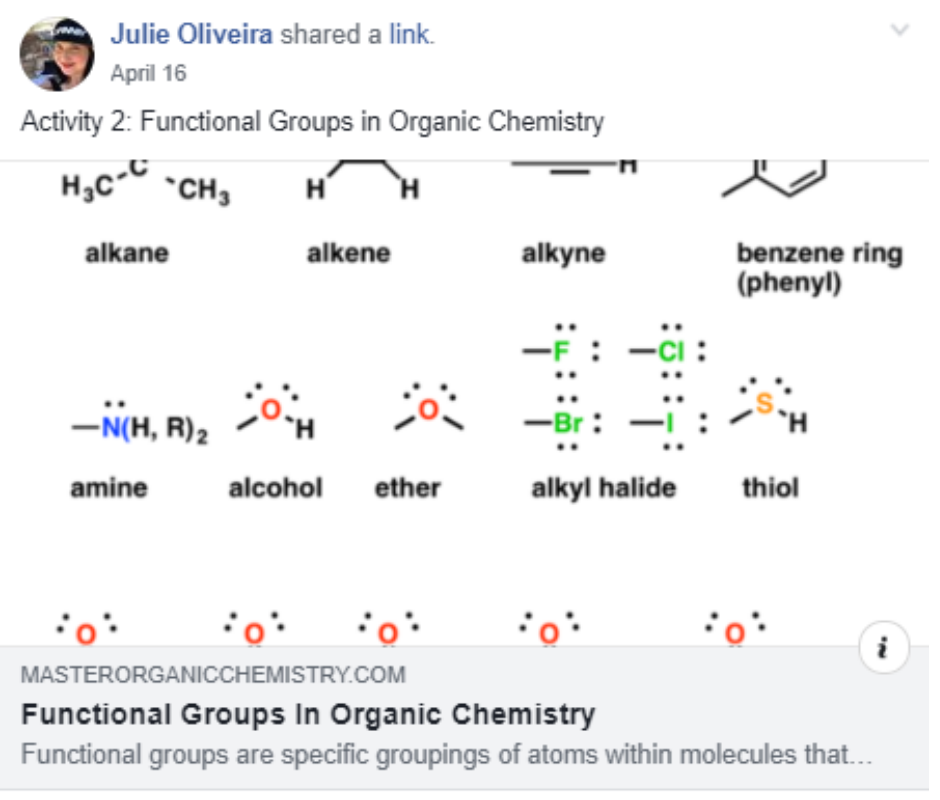

13 Comments Seen by 14

Figura 3 - Atividade Sobre Grupos Funcionais.

Fonte: https://www.masterorganicchemistry.com/2010/10/06/functional-groups-organic-chemistry/

Na sequência foram postadas perguntas sobre o álcool combustível na perspectiva do vídeo nos comentários da postagem, como "What is methanol produced from?" e novamente os estudantes puderam escolher se responderiam em língua inglesa ou em língua portuguesa, tendo todos optado por responder em português. Eles também foram encorajados a criar perguntas para os colegas, mas se limitaram a responder as que já haviam sido criadas pela professora.

A terceira parte da aula 2 envolveu o estudo de ésteres como flavorizantes. Os estudantes iniciaram lendo um pequeno texto sobre o assunto Ester, chemical compound (Figura 4). Após foi realizada uma atividade que relacionava os ésteres com os sabores e aromas correspondentes, na forma de flashcards digitais ${ }^{4}$, que são pequenos cartões em que se relacionam conceitos ou se organiza um sistema de perguntas e respostas para memorizar um conteúdo. Estes flashcards estão disponíveis em uma plataforma online chamada Quizlet, de acesso e conteúdo gratuitos.

${ }^{4}$ QUIZLET. Esters as Flavoring Agents Flashcards. Disponível em: https://quizlet.com/127695458/estersflavoring-agents-flash-cards/-cards/: Acesso em: 16 abr. 2019.

V. $17 \mathrm{~N}^{\circ}$ 3, dezembro, 2019

DOI: 
Activity 4: Esters as flavoring agents

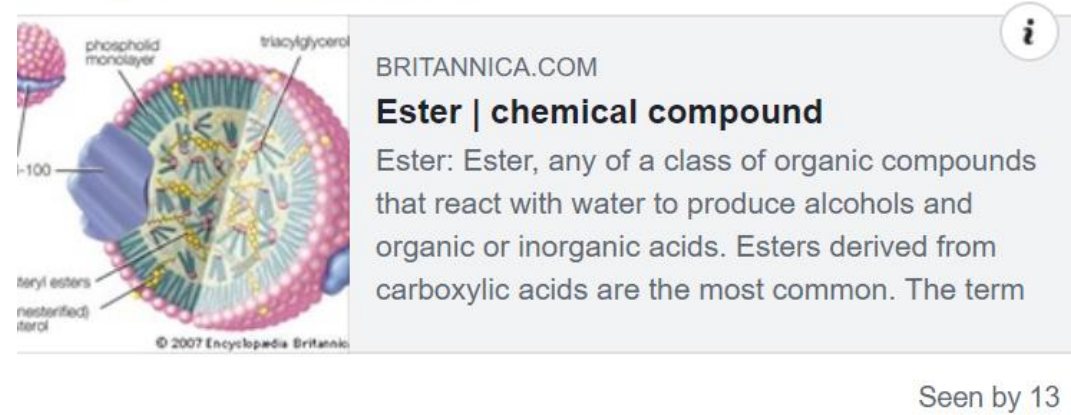

Figura 4 - Atividade Sobre Ésteres como Flavorizantes.

Fonte: https://www.britannica.com/science/ester-chemical-compound

A última atividade da aula 2 foi um teste online também sobre os ésteres como flavorizantes na plataforma Quizlet $^{5}$.

Após o término das atividades da aula 2, os estudantes foram provocados a participar de uma atividade EAD para o estudo dos aminoácidos.

As atividades para o estudo dos aminoácidos foram iniciadas com a proposição de leitura do material "Essential Amino Acids: Definition, Benefits and Food Sources"6", com o link nos comentários para um texto complementar, para os flashcards e para acesso ao teste online ${ }^{7}$.

Com base na participação dos estudantes nas atividades e na observação do comportamento e comentários que os estudantes fizeram durante a realização das atividades, foi possível constatar que a utilização do Facebook como AVA foi bem-sucedida. As principais dificuldades foram em relação à língua inglesa, pois a turma possuía diferentes níveis de conhecimento acerca desta. Houve grande interação entre eles na tentativa de ajudar os colegas e assim 100\% dos estudantes realizaram 100\% das atividades propostas.

Com o objetivo de avaliar a utilização do Facebook como AVA no ensino de química orgânica foi aplicado um questionário logo após a realização de cada aula digital, por meio do qual foi possível analisar as percepções dos estudantes em relação às atividades desenvolvidas, aos conteúdos dessas atividades e principalmente, em relação à contribuição do Facebook como AVA.

Após a aula digital 1,72,7\% dos estudantes responderam que acharam interessante e gostariam de utilizar o Facebook para fins educativos com mais frequência nas disciplinas, 18,2\% responderam que para eles é indiferente e apenas 9,1\% respondeu que achou difícil. Quando perguntado, o participante que achou difícil estava referindo-se à dificuldade com a língua inglesa e não com a interface da rede social nem com os conteúdos de química orgânica abordados. Na aula 2 aumentou a participação de estudantes e aumentou a porcentagem dos que acharam interessante utilizar o Facebook como AVA de 90,9\% para $92,3 \%$, nenhum ficou indiferente e apenas 1 continuou respondendo que achou difícil.

${ }^{5}$ QUIZLET. Test. Disponível em: https://quizlet.com/127695458/test Acesso em: 16 abr. 2019.

6 KUBALA, Jillian. Essential Amino Acids: Definition, Benefits and Food Sources. Disponível em: https://www.healthline.com/nutrition/essential-amino-acids. Acesso em: 16 abr. 2019.

${ }^{7}$ QUIZLET. Amino Acids Flashcards. (https://quizlet.com/2855398/amino-acids-structure-to-full-name-flashcards/). Acesso em: 16 abr. 2019.

V. $17 \mathrm{~N}^{\circ}$ 3, dezembro, 2019 RENOTE

DOI: 
Em relação à compreensão dos conteúdos desenvolvidos nas aulas digitais por meio do Facebook, 63,6\% dos participantes da aula 1 (7 estudantes) responderam que conseguiram compreender os conteúdos e acreditam que houve aprendizado, enquanto os outros $36,4 \%$ (4 estudantes) declararam ter compreendido parcialmente. Nenhum dos participantes marcou a opção "não compreendeu e não houve aprendizado". Na segunda aula aumentou a porcentagem dos estudantes que conseguiram compreender para 69,2\% (9 estudantes) e os outros 4 estudantes $(30,8 \%)$ ainda responderam que compreenderam parcialmente conforme defendido por Miranda (2007) que diz que "a introdução de novos meios tecnológicos no ensino irá produzir efeitos positivos na aprendizagem” (Miranda, 2007, p.42).

Quando perguntados sobre o uso do Facebook nos processos de aprendizagem da disciplina logo após a aula 1, 8 estudantes apresentaram respostas positivas, dizendo que gostaram, que acharam interessante e 1 deles apontou que o Facebook "introduz aprendizado em um meio geralmente ligado ao lazer", 3 dos estudantes apresentaram respostas negativas, dizendo que acharam "meio complicado", "não tenho certeza se trará algum benefício" e "não foi muito do meu gosto pois uso o Facebook para fins de desopilação do dia a dia corrido". Após a aula 2 todos os 12 estudantes que responderam a pergunta apresentaram respostas positivas em relação ao uso do Facebook como AVA, 1 apenas com a ressalva de que utilizar a língua inglesa nas atividades dificultou o entendimento.

Em relação ao uso do Facebook como AVA, apenas duas respostas foram negativas como "não utilizo muito o Facebook" e "não gosto de utilizar o Facebook, responder perguntas publicamente faz com que outros colegas apenas copiem as respostas". Cabe aqui ressaltar que um dos objetivos das aulas digitais era proporcionar contato com a língua inglesa e a construção coletiva dos saberes, então era esperado que os colegas visualizassem as respostas dos demais, podendo servir como ajuda para aqueles com mais dificuldades em encontrar a resposta correta.

Dos estudantes que participaram das aulas digitais 1 e 2, apenas 1 respondeu que o Facebook não favorece trabalhos colaborativos tanto após a primeira quanto após a segunda aula, sendo que após a segunda aula a porcentagem que respondeu que sim - favorece trabalhos colaborativos - aumentou de $72,7 \%$ para $92,3 \%$.

Ao final do questionário foi destinado um espaço para críticas e/ou sugestões e surgiram respostas como: "achei a iniciativa superinteressante, pois trazer aulas interativas e diferentes para entreter os alunos é um ótimo diferencial em um professor, acho que nosso mundo está se tornando cada vez mais virtual e não temos como escapar disso e devemos nos adaptar". Nas respostas dos questionários, 92,3\% dos participantes avaliaram positivamente os procedimentos de ensino adotados por meio da rede social, ou seja, é possível utilizar o Facebook como AVA, à medida que permite diferentes estratégias de ensino e aprendizagem - como vídeos, textos, imagens, jogos... - e facilita a interação entre os participantes do grupo fechado.

\section{Considerações Finais}

Por meio dessa pesquisa foi possível confirmar o potencial do Facebook para fins educacionais com sucesso. O Facebook ofereceu algumas vantagens quando comparado com as plataformas de apoio ao ensino e aprendizagem tradicionais, como não necessitar de cadastro adicional (todos os estudantes do grupo já usavam a rede social), não necessitar de formação prévia, pois já estavam familiarizados com as funcionalidades oferecidas e, por 
estar mais associado a momentos de lazer, proporcionou descontração e empolgação para os participantes das atividades.

A revisão de literatura demonstrou que a forma mais indicada de se trabalhar com o Facebook como AVA é através de grupos fechados, por este motivo foi a forma adotada neste estudo. A revisão de literatura demonstrou também que os resultados alcançados com as pesquisas internacionais estudadas muito se assemelham aos resultados alcançados nas pesquisas nacionais, por meio das quais foi constatado que os estudantes preferem utilizar o Facebook aos sistemas tradicionais de suporte ao ensino e aprendizagem e que as principais atividades desenvolvidas são: compartilhamento de textos, vídeos, criação de material textual, utilização do chat para troca de informações e leituras com interpretação de textos.

A análise da aceitação das aulas digitais realizadas por meio do Facebook junto aos participantes demonstrou que eles não tiveram nenhum tipo de resistência às atividades propostas, tendo $100 \%$ dos estudantes realizado $100 \%$ das atividades presenciais e $92,3 \%$ respondido que gostariam de utilizar o Facebook para fins educativos com mais frequência. Verificou-se também que a participação deles aumentou da primeira para a segunda aula, não apenas em número, mas também nas respostas positivas às perguntas dos questionários.

Da ATD emergiram categorias que não estavam previamente definidas nas perguntas, como a dificuldade que alguns estudantes tiveram com o conteúdo em língua inglesa. Para o docente também há vantagens no uso do Facebook, podendo acompanhar em tempo real o desenvolvimento das atividades, os comentários, as postagens, compartilhamentos, entre outros. Enfim, todas as experiências dos estudantes e do professor podem ser compartilhadas e acessadas a qualquer momento no próprio ambiente escolar ou fora dele, propiciando maior aproximação entre professor-estudante e estudante-estudante em torno do conteúdo estudado. Nesse sentido, entende-se que as experiências educacionais mediadas pelas TIC, especialmente por uma rede social como o Facebook, contribuem com a melhoria dos processos de ensino e aprendizagem.

\section{Referências}

CAMPOS, H; SAMPAIO, S. A Facebook page to share didactic resources: a case study. In: 10th annual International Conference of Education, Research and Innovation. Proceedings of ICERI2017 Conference. Seville, Spain: November, 2017. p 6267-6276.

CASTELLS, M. A sociedade em rede. São Paulo: Paz e Terra, 1999. v. 1

CATÃO, S. N. O Facebook como recurso didático: ferramenta pedagógica utilizada no ensino de Química. 2014. 35 p. Trabalho de conclusão de Curso (Especialização em Fundamentos da Educação: Práticas Pedagógicas Interdisciplinares) - Universidade Estadual da Paraíba. Campina Grande, 2014.

GIL, A. C. Métodos e técnicas de pesquisa social. 5. ed. São Paulo: Atlas, 1999.

MIRANDA, G. L. Limites e possibilidades das TIC na educação. Revista de Ciências da Educação. Porto Alegre, n 3. maio/ago 2007. p 41-50.

MORAES, R; GALIAZZI, M. C. Análise textual discursiva: Processo reconstrutivo de múltiplas faces. Revista Ciência \& Educação, Bauru. v. 12, n. 1, p. 117-128, 2006. 
PORTO, C; SANTOS, E. (orgs). Facebook e educação: publicar, curtir, compartilhar. Campina Grande: EDUEPB, 2014.

SCHROEDER, J; GREENBOWE, T. J. (2009). The Chemistry of Facebook: Using Social Networking to Create an Online Community for the Organic Chemistry Laboratory. Innovate: Journal of Online Education: Vol. 5: Iss. 4, Article 3.

SILVA, J. M; MARTINS JÚNIOR, F. R. F. Desenvolvimento docente e monitoria de professores em formação com apoio duma rede social: a experiência de licenciandos em Ciências com o Facebook. Educação, Formação \& Tecnologias, v. 10, n. 1. 59-73, 2017. 